\title{
The Atomic Structure of Lattice-Mismatched Si/Ge Bulk Heterostructures by Using the Density Functional Theory
}

\author{
S. Dalgic, and S. Guler \\ Physics Department, Faculty of Science, Trakya University, 22100, Edirne (TURKEY)
}

\begin{abstract}
We present results for the atomic structure of lattice-mismatched Si/Ge bulk heterostructures by first-principles calculations based on the density functional theory. We also analyze the ground state, lattice constant, total energy, and average effective potential of Si/Ge bulk heterostructures which are modeled as monolayer and bilayer in the (001) direction investigated by using the plane wave self consistent field. Finally, an obvious difference between effective potentials of $\mathrm{Si}$ and $\mathrm{Ge}$ is observed in the result of calculations. It has been useful in the making of quantum wells of materials such as Si and Ge. Therefore, it has an important role in the invention of electronic and opto-electronic devices.
\end{abstract}

\section{Introduction}

Silicon and germanium are very important semiconductor materials of group IV. Silicon and germanium have four valence electrons, so silicon and germanium have similar chemical and physical properties. Si and Ge have lattice constant $a_{\mathrm{Si}}=10.265$ a.u. and $a_{\mathrm{Ge}}=$ 10.622 a.u; respectively. While they keep $\left|\frac{a_{G e}-a_{5 i}}{\alpha_{5 t}}\right| \geqslant 1 \%$

these materials are called lattice-mismatched.

The simplest case of lattice-mismatched heterojunctions is $\mathrm{Si} / \mathrm{Ge}[1]$, which is an example of an isovalent homopolar interface. The offset $\mathrm{V}_{\text {eff }}$ is about $0.44 \mathrm{eV}$ and $\mathrm{Ge}$ is higher than $\mathrm{Si}$ for the configuration corresponding to a substrate made of $50 \%-50 \%$ alloy. The Si/Ge (001) heterojunction has been choosen by a number of investigators as a prototypical material system in which to study the effects of strain on band offset values $[2,3]$. The $\mathrm{Si} / \mathrm{Ge}$ material system is especially appropriate for this type of study, because of the large lattice mismatch (4.18\%) between $\mathrm{Si}$ and Ge and because conditions under which coherently strained epilayers can be grown are well known [4,5]. In addition, theoretical calculations of the $\mathrm{Si} / \mathrm{Ge}(001)$ valence-band offset have been performed that explicitly incorporate the effects of strain;[2] these calculations have been confirmed by several experimental results[6,7] indicating that strain strongly influences the value of the valence band-offset. The Si/Ge interface is also of great technological interest because of the possibility of integrating devices utilizing $\mathrm{Si} / \mathrm{Si}_{1-\mathrm{x}} \mathrm{Ge}_{\mathrm{x}}$ heterojunctions directly into existing Si-based structures. The behavior of $\mathrm{Si} / \mathrm{Si}_{1-\mathrm{x}} \mathrm{Ge}_{\mathrm{x}}$ heterostructure devices depends critically on the values of the conduction- and valence-band offsets[8].

Density functional theory (DFT) is one of the most widely used methods for "ab initio" calculations of the structure of atoms, molecules, crystals, surfaces and their interactions[9]. Also DFT is a ground-state theory in which the emphasis is on the charge density as the relevant physical quantity. DFT has proved to be highly successful in describing structural and electronic properties in a vast class of materials ranging from atoms and molecules to simple crystals to complex extended systems (including glasses and liquids). Furthermore DFT is computationally very simple. For these reasons DFT has become a common tool in first-principle calculations aimed at describing or even predicting properties of molecular and condensed matter systems[10]. While DFT in principle gives a good description of ground state properties, practical applications of DFT are based on approximations for the so-called exchange-correlation potential. The exchangecorrelation potential describes the effects of the Pauli principle and the Coulomb potential beyond a pure electrostatic interaction of the electrons. Possessing the exact exchange-correlation potential means that we solved the many-body problem exactly, which is clearly not feasible in solids. 
In this paper, we report the atomic structure of lattice-mismatched $\mathrm{Si} / \mathrm{Ge}$ bulk heterostructure by first principle calculations based on DFT and discuss the average effective potential of $\mathrm{Si} / \mathrm{Ge}$ system

\section{Theory}

The quantum mechanical properties of a system of $\mathrm{N}_{\mathrm{el}}$ interacting electrons in the external potential of the nuclei can be obtained by solving the many-body Schrodinger equation:

$$
\hat{H} \psi\left(r_{1}, \ldots, r_{N_{n}}\right)=E \psi\left(r_{1, \ldots}^{r_{1}, r_{N}}\right)
$$

with the following Hamiltonian:

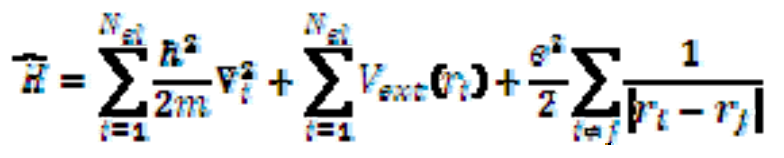

$$
\text { (2) } E_{L}=\frac{1}{2} \int \frac{n\left(\theta_{1}^{r}\right) n\left(\theta_{2}^{r}\right)}{\left|r_{1}-r_{2}^{r}\right|} d^{s} r_{1} d^{s} r_{2}
$$

where $\mathbf{r}_{\mathrm{i}}$ is the position of electron $\mathrm{i}, \mathbf{v}_{i}^{\boldsymbol{z}}$ indicates the laplacian taken with respect to the coordinate ri, while $\mathrm{V}_{\text {ext }}\left(\mathbf{r}_{\mathrm{i}}\right)$ is the external potential acting on the electrons and depends parametrically on the nuclear positions. With this choice, in Eq. (2) we can make the substitutions: $\mathrm{e}^{2}=1$, for the square of the electron charge, and $\mathrm{m}=1$, for the electron mass.

DFT was introduced in 1964 by Hohenberg and Kohn[11], who proved a one-to-one correspondence between the ground-state electronic charge density $n(\mathbf{r})$ and the external potential $\mathrm{V}_{\text {ext }}(\mathbf{r})$. Therefore, since the external potential determines also the many-body wavefunction of the ground state, every physical quantity of the system in its ground state can be expressed as a functional of the electronic charge density. We express the total energy as a functional of the electronic density :

$$
\begin{aligned}
& E[n[r]]=\left\langle\psi_{\mathrm{o}}|\hat{H}| \psi_{0}\right\rangle
\end{aligned}
$$

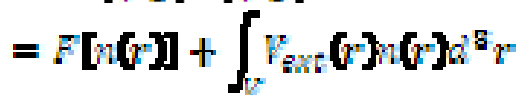

where the integral is done over the whole volume $\mathrm{V}$ of the system. In this equation, $\mathrm{F}[\mathrm{n}(\mathrm{r})]$ is a universal functional of the density and it is given by the expectation value of the kinetic energy and of electrostatic electron repulsion terms on the ground state. The minimization of the functional $\mathrm{E}\left[\mathrm{n}\left(\boldsymbol{r}^{*}\right)\right]$ with respect to the electronic density, with the constraint that the number of electrons $\mathrm{N}_{\mathrm{el}}$ is fixed,

$$
\int_{V} n(x) d d^{8} r=N_{e l}
$$

gives the ground-state total energy and electronic density. If we call $\psi_{\mathrm{i}}(\mathrm{r})$ the single-particle wave-functions, then the kinetic energy of a non-interacting electrons system is:

$$
T_{0}=\sum_{f} f\left(-\frac{1}{2} \nabla^{2} \mid t\right)
$$

where $f_{i}$ is the occupation factor for state i. The density can be written as:

$$
n(x)=\sum_{t} f(t)\left(3^{2}\right.
$$

and is chosen equal to the ground-state electronic density of the interacting electrons system. From $\mathrm{F}[n$ (r)] we can subtract a Hartree-like energy term, which accounts for the classical Coulomb interaction of a spatial charge distribution $n$ (r):

and thus rewrite the universal functional as:

$$
F[n(r)]=T_{0}+E_{H}+E_{e x c}[n(r)]
$$

where $E_{x c}$ is the so-called "exchange-correlation" functional, which contains all the many-body interaction effects not included in the other energy terms. Using the expression of the density given in Eq. (6), the interaction energy associated to the external potential can be rewritten as:

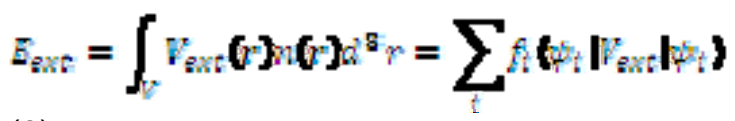

and hence it becomes convenient to minimize the total energy functional,

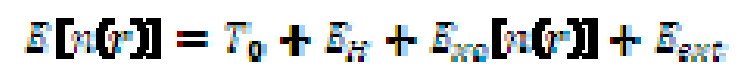

with respect to the single-particle wave-functions $\psi_{i}$ (r). The wave-functions are normalized to one and satisfy the orthogonality constraint,

$$
\left.w_{i}\right)=b_{i, j}
$$

so that the minimization of the total energy functional can be performed through the Lagrange multiplier technique. The solution leads straightforwardly to the Kohn-Sham (KS), equations[12], which read:

$$
\left[-\frac{1}{2} \nabla^{2}+v_{K},(r)\right] \psi_{i}(r)=\tau_{i} \nu_{i}(r)
$$


where the KS potential has been defined as

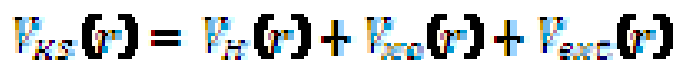

and contains, besides the external potential, the Hartree potential,

$$
V_{H}\left(r^{n}\right)=\int \frac{n\left(r^{\prime \prime}\right)}{\left|\boldsymbol{r}^{n}-\boldsymbol{r}^{r}\right|} d^{3}{ }^{{ }^{\prime}}
$$

and the exchange-correlation potential,

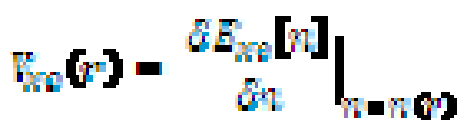

From the solution of the KS equations one obtains the auxiliary single-particle wavefunctions, $\mathrm{i}$, that give the ground-state electronic density through Eq. (6).

\section{Results and Discussion}

The calculation is based on DFT in the local-density approximation. We use a plane wave expansion ab initio Ultrasoft - Pseudopotantial[13] as implemented in the Plane wave self consistent field code (PWscf)[14]. The number of plane waves used for these calcualtions was determinated by an energy cut-off 35 Ryd. We have used a $1 \times 1 \times 2$ mesh(monolayer) and $1 \times 1 \times 4$ mesh(bilayer), corresponding to six $\mathrm{k}$ point that turns out to be sufficient for the accuracy of the results.

$\mathrm{Si} / \mathrm{Ge}$ bulk heterostructure which is modelled as monolayer and bilayer in the (001) direction have been investigated. The lattice constants of these models have been determined and Poisson ratios have been calculated by making optimization in the $\mathrm{z}$ direction. After that we have calculated relaxation of atoms by using the Poisson ratio. In this result of calculation, total energy and the average effective potential of the system have been achieved for monolayer and bilayer Si/Ge.

The atomic positions of monolayer and bilayer have been defined. Bilayer structure has been derived from a two unit cell. Two Si atoms and two unit cell Ge atoms by growthing in the $\mathrm{z}$ direction. Fig. 1(a) and Fig. 1(b) show the monolayer and bilayer $\mathrm{Si} / \mathrm{Ge}$ bulk heterostructure.
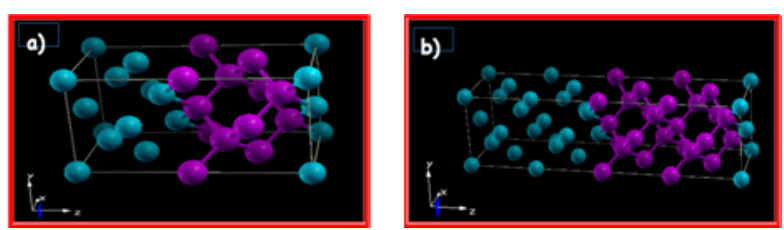

\begin{tabular}{l|cccc}
\hline \hline \multicolumn{5}{c}{ Monolayer } \\
\hline \hline $\mathrm{z}(\mathrm{a})$ & 1.02 & 1.01 & 0.99 & 0.98 \\
$\mathrm{x}=\mathrm{y}(\mathrm{a})$ & 0.9949 & 0.9973 & 1.006 & 1.0050 \\
$\mathrm{E}_{\min }\left(\mathrm{R}_{\mathrm{yd}}\right)$ & -127.453 & -127.456 & -127.456 & -127.454 \\
Posisson ratio & 0.2545 & 0.2670 & -0.2670 & -0.2545 \\
\hline \hline
\end{tabular}

Fig. 1. Bulk heterostructure of Si/Ge system (a)monolayer, (b)bilayer.
Total energy as a function of lattice constant monolayer and bilayer $\mathrm{Si} / \mathrm{Ge}$ bulk heterostructures is presented in Fig. 2(a) and Fig. 2(b). The result of calculation shows that the lattice constants are 10.404 a.u and 10.410 a.u. corresponding to minimum energy for monolayer and bilayer, respectively.
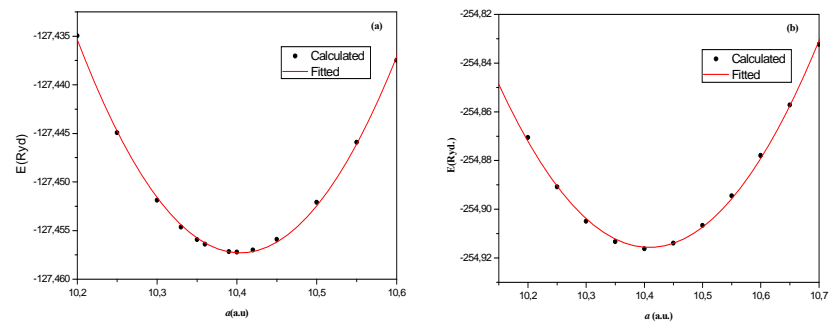

Fig. 2. Total energy as a function of the lattice constant for the monolayer and bilayer Si/Ge bulk heterostructures.

In both systems, $\mathrm{Si} / \mathrm{Ge}$ bulk heterostructure has been obtained as super lattice that grew in the $\mathrm{z}$ direction. The extension of the $\mathrm{Si} / \mathrm{Ge}$ system has been calculated by extending in the $\mathrm{z}$ direction and compressing in the $\mathrm{x}, \mathrm{y}$ direction and also compressing in the $\mathrm{z}$ direction and extending $\mathrm{x}, \mathrm{y}$ direction. According to the calculated Poisson ratio relaxation of the system has been obtained.

Total energy as a function of $\mathrm{x}=\mathrm{y}(\mathrm{a})$ is shown in Fig.3(a) and Fig.3(b) for both systems as is seen in Fig.3, when $\mathrm{z}=0.98 \mathrm{a}, \mathrm{x}=\mathrm{y}(\mathrm{a})$ is found as 1.00509. In addition $\mathrm{z}(\mathrm{a})$, $\mathrm{x}=\mathrm{y}(\mathrm{a})$, minimum energy, and Poisson ratio are listed in the Table 1and Table 2.
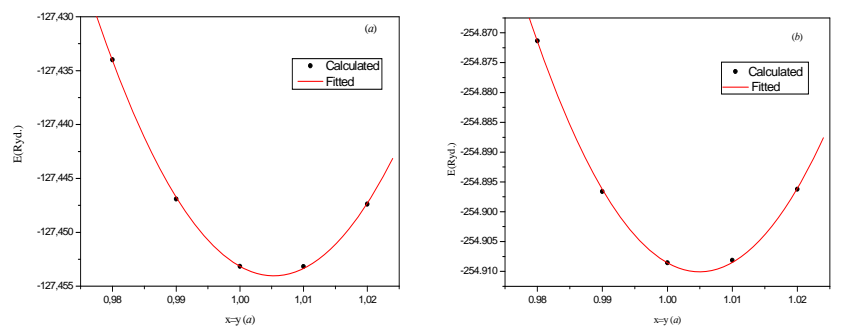

Fig. 3. Total energy as a function of $x=y(a)$ for the monolayer and bilayer Si/Ge bulk heterostructures.

Table 1. Optimization Parameters for monolayer Si/Ge system 
Table 2. Optimization Parameters for bilayer Si/Ge system

\begin{tabular}{l|cclc}
\hline \hline \multicolumn{5}{c}{ Bilayer } \\
\hline \hline $\mathrm{z}(\mathrm{a})$ & 1.02 & 1.01 & 0.99 & 0.98 \\
$\mathrm{x}=\mathrm{y}(\mathrm{a})$ & 0.9944 & 0.9968 & 1.0021 & 1.0050 \\
$\mathrm{E}_{\min }\left(\mathrm{R}_{\mathrm{yd}}\right)$ & -254.908 & -254.914 & -254.914 & -254.910 \\
Posisson ratio & 0.2790 & 0.3150 & -0.2180 & -0.2545 \\
\hline \hline
\end{tabular}

We present in Fig.4(a) and Fig.4(b) the average effective potentials for both $\mathrm{Si} / \mathrm{Ge}$ systems. Figures show clearly that the average effective potentials are obtained from a supercell calculation. The supercell size is enough to reproduce the bulk-like character at both sides of the interface and the effect of the relaxation of the atomic positions at the interface region due to the strain related with the lattice constant mismatch of the Si/Ge[15,16]. An obvious difference between average effective potentials of $\mathrm{Si}$ and $\mathrm{Ge}$ has been observed in the result of calculations. This difference is $0.44 \mathrm{eV}$.
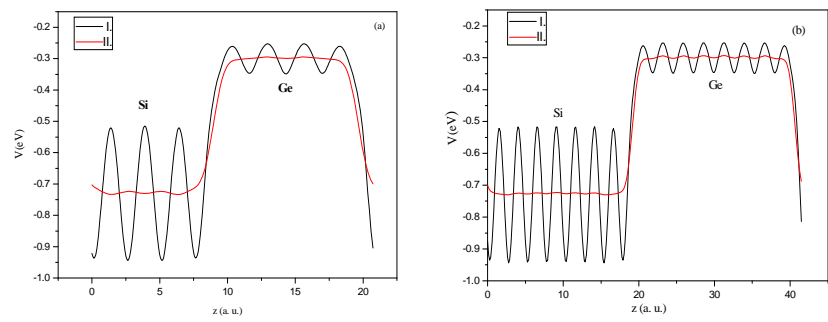

Fig. 4. Average effective potentials for monolayer and bilayer Si/Ge bulk heterostructures.

\section{Conclusions}

We present a theoretical study of the atomic properties of lattice-mismatched $\mathrm{Si} / \mathrm{Ge}$ bulk heterostructures using first principle total energy and average effective potential calculations based on the density functional theory.

It is clear that the difference between average effective potentials of $\mathrm{Si}$ and Ge has been observed. This is very significant because of quantum wells of materials such as Si and Ge. For this reason, it has an important role in the invention of electronic and opto-electronic devices.

\section{References}

1. L. Colombo, R. Resta and S. Baroni Phys. Rev. B44, 5572 (1991)

2. C. G. Van de Walle and R. M. Martin, Phys. Rev.B34, 5621 (1986)

3. G. P. Schwartz, M. S. Hybertsen, J. Bevk, R. G.

Nuzzo, J. P. Mannaerts and G. J. Gualtieri, Phys. Rev. B39, 1235 (1989)

4. J. H. Van der Merwe, J Appl. Phys. 34, 123 (1963)

5. R. H. Miles, T. C. McGill, P. P. Chow, D. C. Johnson,
R. J. Hauenstein, C. W. Nieh and M. D. Satrathman, Appl. Phys. Lett. 52. 916 (1988)

6. W. -X. Ni, J. Knall and G. V. Hansson, Phys Rev. B 36, 7744 (1987)

7. E. T. Yu, E. T. Croke, T. C. Mc Gill and R. H. Miles, Appl. Phys. Lett. 56, 569 (1990)

8. E. T. Yu, J. O. Mc Caldin and T. C. Mc Gill, Solid State Physics vol 46, ed H. Ehrenreich and D. Turnbull (Boston, MA: Academic) p 1 (1992)

9. R. G. Parr and W. Yang, Density Functional Theory of Atoms and Molecules (Oxford, 1989)

10. A. A. Katsnelson, Computational methods in condensed matter: electronic structure, American Institute of Physics (New York, 1992)

11. P. Hohenberg and W. Kohn, Phys. Rev. 136, 864 (1964)

12. W. Kohn and L. J. Sham, Phys. Rev. 140, 1133 (1965)

13. D. Vanderbilt Phys. Rev. B 328412 (1985)

14. S. Baroni, A. Dal Corso, S. de Gironcoli and P. Giannozzi, http://www.pwscf.org

15. M. Peressi, S. Baroni, R. Resta, A. Baldereschi. Phys. Rev. B 43, 7347 (1991)

16. Peressi M., Binggeli N., and Baldereschi A., J. Phys. D: Appl. Phys. 31, 1273 (1998) 